\title{
Biological significance and synthetic approaches for 1,3,4-Thiadiazoles: A Review
}

Authors \& Affiliation:

Gali Pushpa Raju, ${ }^{1, *}$ Sailaja Gugulothu ${ }^{2}$

Khasim Sharif Syed ${ }^{3}$

${ }^{I}$ Department of Chemistry, C.R. College, Chilakaluripet, Guntur Dist., AP-India

${ }^{2}$ Singareni Colleries Women's

Degree College, Bhadradri

Kothagudem-507101, TelanganaIndia

${ }^{3}$ Departmentt. of Chemistry, Dr.SRK Govt Arts College, Affiliated to

Pondicherry Central University,

Yanam, 533464, India

\section{Corresponding Author}

\section{Gali Pushpa Raju}

Email: pushparaju5gali@gmail.com

(C) 2019.The Authors. Published under Caribbean Journal of Science and Technology

ICNN N700 2757

\section{Abstract:}

In this article the authors presented a short review of literature survey on the synthetic approaches for the synthesis of thiadiazoles and their derivatives. Further, various biological activities of these derivatives were also summarized.

Keywords: Thiadiazole, synthesis, pharmacological activity

\section{Introduction}

Thiadiazole is a prevalent biologically active scaffold in heterocyclic compounds having five membered ring consisting of 2 nitrogen atoms, 2 carbon atoms, 1 sulphur atom, and 2 double bonds. Thiadiazole moiety behaves as "hydrogen binding domain" and "two-electron donor system". As shown in Figure 1, 4 isomers of thiadiazoles (Figure 1) are possible based on the nitrogen atom position in the ring. Among these isomers, 1,3,4-thiadiazoles have been studied more than other isomers owing to possessing versatile biological activities. ${ }^{1}$<smiles>c1csnn1</smiles>

$1,2,3-$<smiles>c1ncsn1</smiles>

$1,2,4-$<smiles>c1cnsn1</smiles>

$1,2,5-$<smiles>c1nncs1</smiles>

$1,3,4-$
Figure 1

1,3,4-Thiadiazoles are more prone to nucleophilic attack due to the presence of 2 ' $\mathrm{N}$ ' in the ring allowing substitutions into 2 ' or 5' positions of the ring to afford wide range of analogues. When treated with aqueous base, ring cleavage can occur, whereas, in acid solutions, the ring has more stability. Thus possessing these properties, 1,3,4-dithiadiazoles derivatives are applied widely in pharmacological, agricultural and material chemistry. ${ }^{2}$

\section{Biological significance of 1,3,4-thiadiazoles}

Several 1,3,4-thiadiazoles are found to have characteristic drug activity in different biological evolution studies, makes the medicinal chemists to concentrate over the synthesis and biological evolution of novel 1,3,4-thiadiazole derivatives. 
The following are a few examples of the 1,3,4-thiadiazole derivatives consists of biological significance.

\subsection{Antimicrobial activity}

Hussain etal $^{3}$ synthesized 4-amino phenol derived 1,3,4-thiadiazoles (Figure 2) which showed promising antimicrobial activity.

\subsection{Anti-Fungal activity}

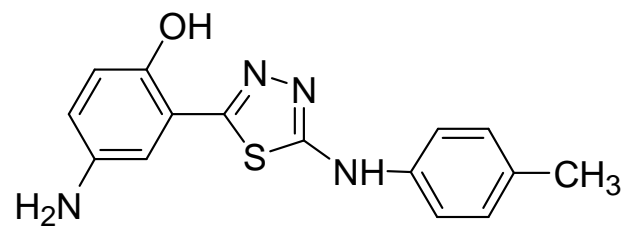

Figure 2

Jun-Chen $e$ t $a l^{4}$ prepared 3,4,5-trimethoxy derived 1,3,4-thiadiazoles (Fig. 3) and screened for antifungal activities, in vitro.<smiles>[R]S(=O)(=O)c1nnc(-c2cc(OC)c(OC)c(OC)c2)s1</smiles>

$$
\begin{aligned}
& \mathrm{R}=(\mathrm{a})=\mathrm{H} 2 \mathrm{C}=\mathrm{CH}-\mathrm{CH} 2 \\
& (\mathrm{~b})=\mathrm{O} 2 \mathrm{~N}-\mathrm{C} 6 \mathrm{H} 6-\mathrm{CH} 2- \\
& (\mathrm{c})=\mathrm{C} 6 \mathrm{H} 6-\mathrm{CH} 2 \\
& \text { ( d })=\mathrm{CH} 3-
\end{aligned}
$$

Figure 3

\subsection{Anticonvulsant activity}

Gupta et $a l^{5}$ have synthesized wide range of 3-aryl amino/amino-4-aryl-5-imino-D2-1,2,4-thiadiazoline derivatives (Fig. 4) and evaluated successfully for their anti-convulsant properties,

\subsection{Anti-inflammatory activity}<smiles>Cc1ccc(-n2c(N)nsc2=N)cc1</smiles>

Sharma et al. ${ }^{6}$ synthesized 2-amino-5-sulfanyl-1,3,4-thiadiazole derivatives which were exploited as selective inhibition of COX-2 enzyme (Fig. 5).<smiles>NS(=O)(=O)c1ccc(Nc2nnc(Sc3ccc(F)cc3)s2)cc1</smiles>

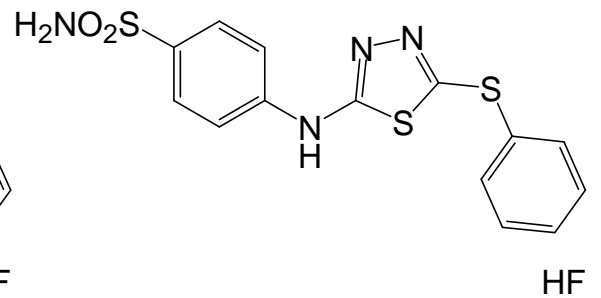

\subsection{Antileishmanial activity}

Figure 5

Ram et $a l^{7}$ prepared several analogues of 2,4 disubstituted 1,3,4 thiadiazole (Fig. 6) and evaluated for antileishmanial activity, in vitro. 


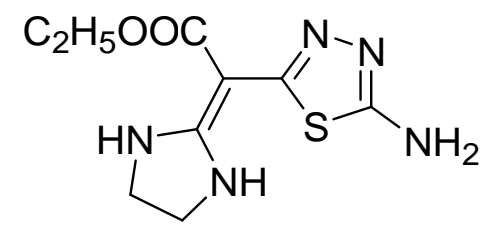

\subsection{Cytotoxic activity}

\section{Figure 6}

Matysiak et $\mathrm{al}^{8}$ synthesized a new series of $\mathrm{N}$-substituted 2-amino-5-(2,4-dihydroxyphenyl)-1,3,4thiadiazoles which showed excellent antiproliferative activities against human cancer cell lines (Fig. 7).<smiles>[R]Nc1nnc(-c2ccc(O)cc2O)s1</smiles>

$\mathrm{R}=$ aryl,alkyl.

Figure 7

\subsection{Antitubercular activity}

Chitra $e t a l^{9}$ synthesized 3-heteroarylthioquinoline derivatives of 1,3,4-thiadiazole and screened their in vitro antimycobacterial activity (Fig. 8).<smiles>[R]c1ccc(-c2nc3ccccc3c(-c3ccccc3)c2Sc2nnc(C)s2)cc1</smiles>

$\mathrm{R}=\mathrm{Cl}, \mathrm{Br}$

Figure 8

\section{Existing Synthetic Approaches}

Abdelwahed et $a l^{10}$ has reported synthesis of thiadiazoles derivatives (Scheme 13) by treatment of hydrazonoyl halides $\mathbf{1}$ with methyl 2-(1-ferrocenylethylidene)hydrazine carbodithioate $\mathbf{8}$ in boiling ethanol/ dimethylformamide in the presence of triethylamine under reflux afforded compound $\mathbf{7}$ as shown in below Scheme 1 . 

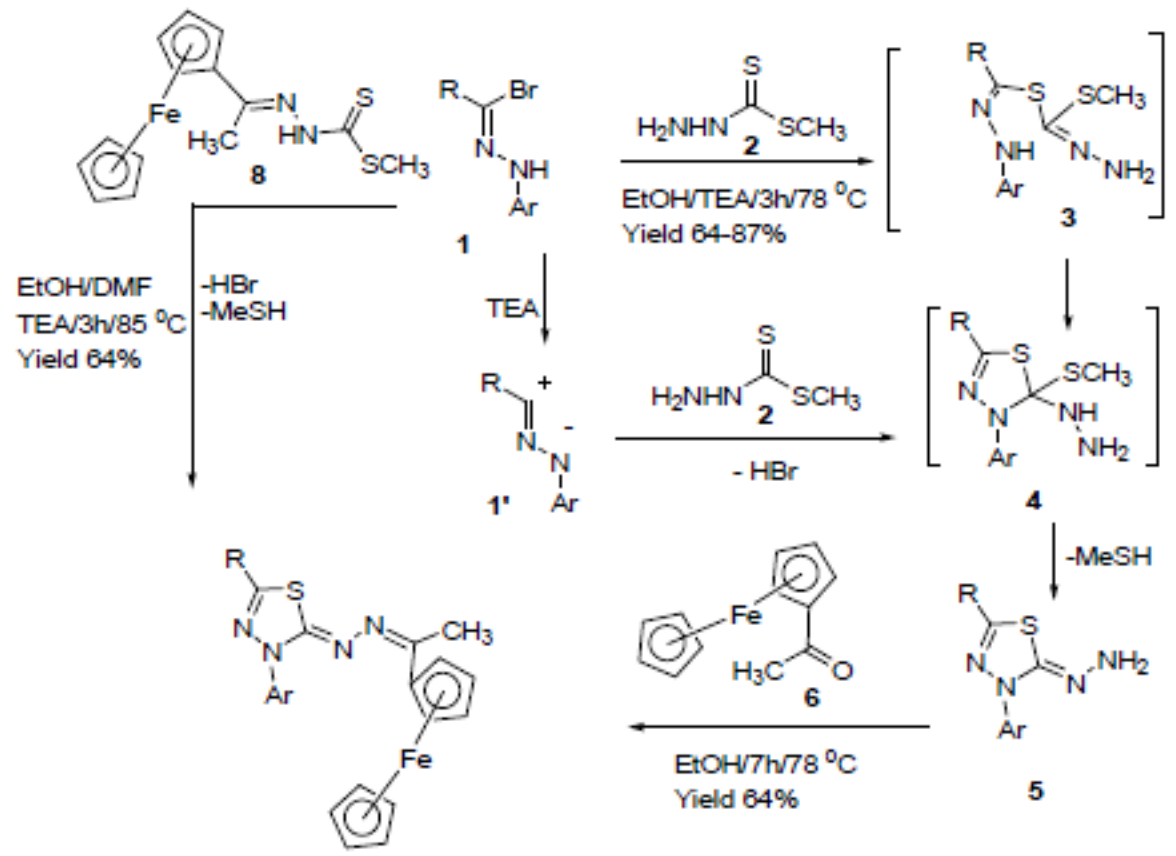

1 ( A

Yield $64-87 \%$

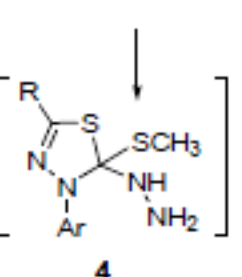

7

R: a, 4- $\mathrm{CH}_{3} O \mathrm{CC}_{6} \mathrm{H}_{4} ; \mathrm{b}, 4-\mathrm{CH}_{3} \mathrm{C}_{6} \mathrm{H}_{4} ; \mathrm{c}, \mathrm{C}_{6} \mathrm{H}_{5} ; \mathrm{d}, 4-\mathrm{ClC}_{6} \mathrm{H}_{4} ; \mathrm{e}, 4-\mathrm{BrC}_{6} \mathrm{H}_{4}$

Ar: $4-\mathrm{BrC}_{6} \mathrm{H}_{4}$

\section{Scheme 1}

Ameen Ali Abu-Hashem et a $1^{11}$ prepared wide range of 1,3,4-thiadiazoles, 1,3,4-thiadiazines derivatives and exhibited higher antimicrobial activity as shown in Scheme 2.<smiles>[R]Nc1nn2c(=O)c3c(C)c(C(C)=O)sc3nc2s1</smiles> 
Tayade and Waghmare et $a l^{12}$ developed one-step preparation of of (2E)-1-[4-(3-substitutedimino-1,2,4dithiazolo) aminophenyl] -3- (3,4- dimethoxyphenyl) prop-2-en-1-one in the presence of $\mathrm{Br}_{2} / \mathrm{CHCl}_{3}$ (Scheme 3).<smiles>[R2]NC(=S)NC(=S)Nc1ccc(C(=O)/C=C/c2ccc(OC)c(OC)c2)cc1</smiles><smiles>COc1ccc(/C=C/C(=O)c2ccc(Nc3nc(=[Pt])ss3)cc2)cc1OC</smiles>

Scheme 3

Naskar et $a l^{13}$ synthesized 2-amino-5-aryl-1,3,4-thiadiazoles by oxidative cyclization of $\begin{array}{lllll}\text { thiosemicarbazones } & \mathrm{FeCl}_{3} & \text { catalyst } & \text { (Scheme }\end{array}$<smiles></smiles><smiles>NNC(N)=S</smiles><smiles>CC(C)O</smiles><smiles>NC(=S)N/N=C\c1ccccc1</smiles>
cyclization<smiles>[R7]c1ccccc1-c1nnc(N=Cc2ccccc2)s1</smiles><smiles>[R2]c1ccccc1C(=O)c1ccccc1-c1nnc(N)s1</smiles>

Scheme 4

Abdel Rahman et $_{\text {al }}{ }^{14}$ synthesized varying range of substituted imidazo [2,1-b]-1,3,4- thiadiazoles, substituted 1,3,4thiadiazolo[3,2-a] pyrimidines and substituted thioureas. Most of the tested compounds exhibited potent cytotoxicity. 
<smiles>[R]c1cccc(-c2cn3nc(-c4ccc(C=[SnH]c5ccccc5)cc4)sc3n2)c1</smiles><smiles>N=c1cc(N)n2nc(-c3ccc(Br)cc3)sc2n1</smiles>

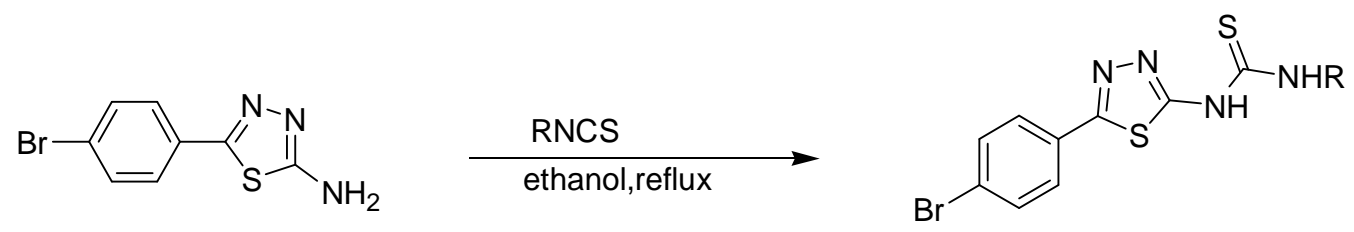

\section{Scheme 5}

Mahendra Singh et $a l^{15}$ has reported the synthesis of thiadiazole derivative from morpholine and ethyl acetoacetate (Scheme 6).

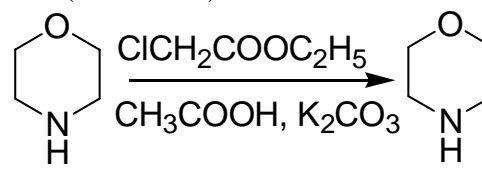

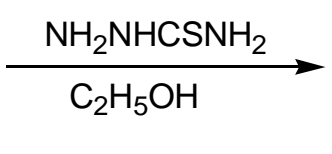<smiles>N[14CH2]NC(=O)OCN1CCOCC1</smiles><smiles>[3H][AsH3]</smiles><smiles>[R2]C([R])=Nc1nnc2n1CCOCC2</smiles><smiles>CC(=O)OCC(=O)O</smiles><smiles>Nc1nc2c(s1)CN1CCOCC21</smiles>

\section{Scheme 6}

Khedr et $\mathrm{l}^{16}$ has synthesized thiadiazole derived Schiff base ligands as shown in Scheme 7.<smiles>Nc1nnc(-c2cc[se]c2)s1</smiles>

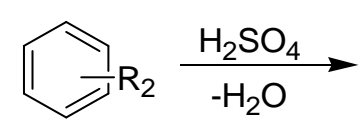<smiles>C1=Cc2ccccc2/C1=N/c1nnc(-c2ccccc2)s1</smiles>

\section{Scheme 7}

\section{Conclusion:}

In conclusion, brief compilation on synthetic approaches and biological relevance of thiadiazole and its derivatives was performed and we do strongly hope that, this short report will be a valuable addition in the said field as well interest to the synthetic and medicinal chemists.

\section{Acknowledgements}

The authors are thankful to C.R.College, Chilakaluripet, Guntur Dist. For constant encouragement. 


\section{Conflict of interest statement}

The authors declare no conflict of interest.

\section{References:}

1. Bhawna. S.; Amita.V.; Sunil. P.; Upendra. K. S.; Int. J.Med. Chem. 2013, 2013, 348948.

2. Hu. Y.; Li. C.Y.; Wang. X.M.; Yang. Y.H.; Zhu. H.-L.; Chem. Rev. 2014, 114, 5572.

3. Sabir. H.; Jyoti. S.; Mohd. A.; Int.J. ChemTech Res. 2011, 3, 1392 Acid; E-J. Chem. 2008, 5, 963.

4. Cai. J C.; Bao-An. S.; Song. Y.; Guang-Fang. Xu.; Pinaki S. Bhadury, Lin-Hong Jin, De-Yu Hu, Qian-Zhu Li, Fang Liu, Wei Xue, Ping Lu and Zhuo Chen; Bioorg. Med. Chem. 2007, 15, 3981.

5. Gupta. A.; Mishra.P; Kashaw.S.K.; Kashaw.V.; Eur. J. Med. Chem. 2008, 43, 749.

6. Rajesh. S.; Jitendra. S. S. C. C.; Acta Pharm. 2008, 58, 317.

7. Da Silva. EF; Canto-Cavalheiro. MM; Braz.VR.; Cysne-Finkelstein. L.; Leon. LL; Echevarria. LA.; Eur. J. Med. Chem. 2002, 37, 979.

8. Matysiak. J.; Opolski.A.; Bioorg. Med. chem. 2006, 14, 4483.

9. Chitra. S.; Paul. N.; Muthusubramanian. S.; Manisankar. P.; Yogeeswari. P.; Sriram. D.; Eur J Med Chem. 2011, 46, 4897.

10. Abdelwahed. R.; Sayed.; Shar Saad. Al.; Molecules. 2017, 22, 336.

11. Ali Abu-Hashem. A.; Faty. R.A.M.; Curr. Org. Syn. 2018, 22, 1161

12. Tayade. DT.; Waghmare. S.A.; J Chem Pharm Res. 2016, 8934.

13. Naskar. A.; Singha. T.; Guria. T.; Singh. J.; Kumar. AB.; Maity. TK.; Int J Pharm Sci. 2015, 7, 397.

14. Rahman. A.; Shakya. A.K.; Wahab. S.; Ansari. N.; Bulg. Chem Commun. 2014, 46, 750.

15. Mahendrasinh. M. R.; Hemul. P.V.; Lata. MR.; Naynika. K.P.; Int J Res Chem Environ. 2013, 3, 9.

16. Abdalla. MK.; Hadi. M.M.; Int J Electrochem Sci. 2012, 10074. 